\title{
Who should be guiding engineering education?
}

\author{
Jason Foster, University of Toronto
}

\begin{abstract}
It seems to be almost an axiom within the engineering education community that the needs of industry should guide the engineering curriculum. On occasion other potential guides, such as the economy, innovation, or the engineering profession are proposed and debates about which guide to follow are undertaken. Thus far in the debate, discussion of the role and responsibilities of such guides appears absent. This paper outlines a number of the more recent discussions of who should guide engineering education, proposes a model to assess the suitability of a potential guide, and asserts that neither industry nor the engineering profession are suitable guides.
\end{abstract}

